EPJ Web of Conferences 60, 15003 (2013)

DOI: $10.1051 /$ epjconf $/ 20136015003$

(C) Owned by the authors, published by EDP Sciences, 2013

\title{
Studies of charmless B decays
}

\author{
Cibrán Santamarina Ríos ${ }^{1, a}$ on behalf of the LHCb collaboration \\ ${ }^{1}$ Departamento de Física de Partículas, Universidade de Santiago de Compostela, Santiago de Compostela, Spain
}

\begin{abstract}
A non-exhaustive review of the analysis of charmless decays of $B$-mesons is presented. Particular emphasis is made in the $B_{s}^{0} \rightarrow \phi \bar{K}^{* 0}$ mode whose discovery and first determination branching fraction and angular parameters were reported for the first time at the LHCP 2013 conference.
\end{abstract}

\section{Introduction}

The study of particle physics aims at discover the elementary particles, their properties and the nature of their interactions. In this context it is known the existence of six quarks, organized in three families, that the Standard Model (SM) describes as stable under the effect of the strong and electromagnetic interactions. Within this theory only the weak interaction allows quark flavour transitions, being the most common the $d \rightarrow u$ transition that is at the origin of the $\beta$ decay of certain radioactive nuclei.

The weak interaction mediated flavour changing transitions provide the only mechanism that breaks-up the $\mathrm{CP}$ symmetry within the SM. Such transitions are described by the CKM matrix [1]. The hierarchy in the CKM matrix establishes that the most favoured flavour transitions are those that take place between two consecutive families. As a consequence, $b$ quark decays are dominated by transitions into a $c$ quark. The alternatives, leading to lighter quarks in the final state, involve a tree-level decay into a $u$ quark or loop mediated decays into a $d$ or an $s$ quark.

Consequently, two elements concur in charmless $B$ meson decays: first, since they are suppressed with respect to charmed decays, there is less knowledge of experimental magnitudes (partial widths, $\mathrm{CP}$ asymmetries, etc.); second, they have more sensitivity to reveal physics beyond the SM. In particular, loop mediated transitions are presumed to be the best benchmark to explore such effects.

The analyses reported in this document are performed in the context of the $\mathrm{LHCb}$ experiment. The LHCb spectrometer is specially designed to have an acceptance at low angles $([10,300] \mathrm{mrad})$ that benefits from the low angle production of $b \bar{b}$ pairs in proton-proton collisions supplied by the Large Hadron Collider (LHC). The cross section of these pairs is, at a center-of-mass energy of $7 \mathrm{TeV}$, $284 \pm 53 \mathrm{mb}$ [2], a factor of $10^{4}$ higher than the production rate at the $B$-factories. This high production rate of $b$-quarks is ideal for collecting and analyzing events containing $B$ mesons that allow the study of $\mathrm{CP}$ violation and

\footnotetext{
ae-mail: cibran.santamarina @usc.es
}

rare decays. Other studies involving $\Lambda_{b}^{0}$ or charmed particles are also the object of analyses. ${ }^{1}$ The identification of secondary vertices where the $B$-mesons decayed and the nature of the decay daughters requires the experiment to achieve excellent position resolution. In addition, particle identification and capability to trigger on hadronic channels are necessary for the study of charmless hadronic decays. The spectrometer was designed consequently and is functioning according to those requirements [4]. $\mathrm{LHCb}$ has accumulated an integrated luminosity of $37 \mathrm{pb}^{-1}$ in 2010, $1 \mathrm{fb}^{-1}$ in 2011 and $2.2 \mathrm{fb}^{-1}$ in 2012. The results reported here correspond to the final analysis of all, or a large fraction, of the 2011 data.

\section{Direct CP violation in charmless B decays}

\subsection{Direct CP violation in $B_{(s)}^{0} \rightarrow K \pi$ decays}

The most straightforward effect of CP violation is the presence of direct $\mathrm{CP}$ violation. This consists in differences in the partial width of $\mathrm{CP}$ reversed decays with contributions from interfering amplitudes with different weak and strong phases. The canonical example is given by the $B^{0} \rightarrow K^{+} \pi^{-}$ and $\bar{B}^{0} \rightarrow K^{-} \pi^{+}$decays. The discovery of direct CP violation in these decays dates back to 2004 [5]. The magnitude that quantifies the size of $\mathrm{CP}$ violating effects is the $A_{C P}$ asymmetry defined as

$$
A_{C P}\left(B^{0} \rightarrow K^{+} \pi^{-}\right)=\frac{\Gamma\left(B^{0} \rightarrow K^{+} \pi^{-}\right)-\Gamma\left(\bar{B}^{0} \rightarrow K^{-} \pi^{+}\right)}{\Gamma\left(B^{0} \rightarrow K^{+} \pi^{-}\right)+\Gamma\left(\bar{B}^{0} \rightarrow K^{-} \pi^{+}\right)} .
$$

$\mathrm{LHCb}$ has recently determined with the best precision ever $A_{C P}\left(B^{0} \rightarrow K^{+} \pi^{-}\right)$to be $-0.080 \pm 0.007$ (stat) \pm 0.003 (syst) [6].

More important, in this same publication $\mathrm{LHCb}$ also reports a determination of $A_{C P}\left(B_{s}^{0} \rightarrow K^{-} \pi^{+}\right)=0.27 \pm$

\footnotetext{
${ }^{1}$ The inclusive cross section of $c \bar{c}$ pairs at $\sqrt{s}=7 \mathrm{TeV}$ proton-proton collisions is $\sigma(\mathrm{p} \overline{\mathrm{p}} \rightarrow c \bar{c} X)=6.10 \pm 0.93 \mathrm{mb}$. [3] which is twenty times the $b \bar{b}$ productions cross-section.
} 


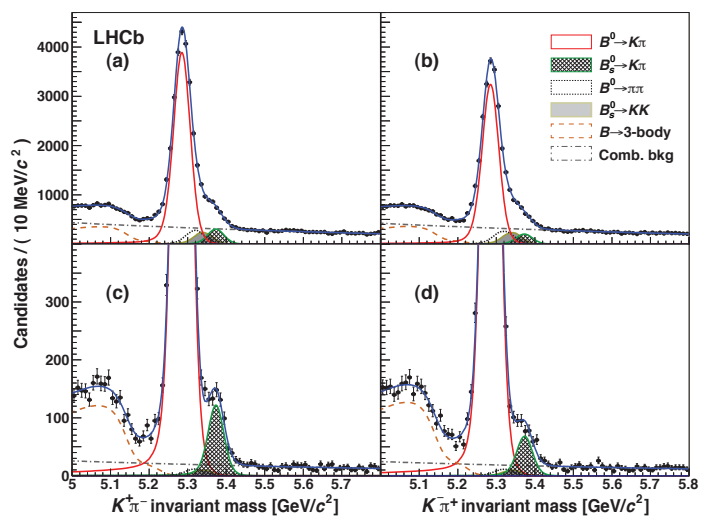

Figure 1. Invariant mass spectra of $K^{+} \pi^{-}$, (a) and (c), and $K^{-} \pi^{+}$, (b) and (d) pairs. Two different selections are shown to optimize the $B^{0}$ (top) and $B_{s}^{0}$ (bottom) signals. The results of an unbinned maximum likelihood fits to a mass model and its main components are overlaid.

0.04 (stat) \pm 0.01 (syst) being the first observation of CP violation in decays of $B_{s}^{0}$ mesons. These two measurements can be combined to provide a consistency check with a SM based prediction for

$$
\Delta=\frac{A_{C P}\left(B^{0} \rightarrow K^{+} \pi^{-}\right)}{A_{C P}\left(B_{s}^{0} \rightarrow K^{-} \pi^{+}\right)}+\frac{\mathcal{B}\left(B_{s}^{0} \rightarrow K^{-} \pi^{+}\right)}{\mathcal{B}\left(B^{0} \rightarrow K^{+} \pi^{-}\right)} \frac{\tau_{d}}{\tau_{s}}=0
$$

given in [7], where $\mathcal{B}$ are the branching fractions of the corresponding decays and $\tau_{d(s)}$ the lifetime of the $B_{(s)}^{0}$ meson. The experimental result of LHCb, $\Delta=-0.02 \pm 0.05 \pm$ 0.04 , shows no evidence of deviation from zero. In this figure the first uncertainty comes from the measurements of the CP asymmetries in $B^{0} \rightarrow K^{+} \pi^{-}$and $B_{s}^{0} \rightarrow K^{-} \pi^{+}$and the second from the input values of the branching fractions and the lifetimes.

\subsection{Direct CP violation in $B^{+}$three-body decays}

As commented above, direct $\mathrm{CP}$ violation arises as a consequence of at least two interfering amplitudes with different weak and strong phases. Whereas the origin of the strong phase in $B_{(s)}^{0} \rightarrow K \pi$ decays is not clear there are theoretical interpretations for phase space $B^{ \pm} \rightarrow h^{+} h^{-} h^{\prime \pm}$ decays, where $h$ and $h^{\prime}$ are either a kaon or a pion. One possible source of the required strong phase is from finalstate hadron rescattering, which can occur between two or more decay channels with the same flavour quantum numbers. This makes the Dalitz analyses of $B^{+}$three-body decays very interesting to understand the interplay between the weak and strong interaction in direct $\mathrm{CP}$ violation.

The $\mathrm{LHCb}$ experiment has explored four different pairs of conjugated modes: $B^{ \pm} \rightarrow \pi^{+} \pi^{-} \pi^{ \pm}, B^{ \pm} \rightarrow K^{+} K^{-} \pi^{ \pm}$[8], $B^{ \pm} \rightarrow \pi^{+} \pi^{-} K^{ \pm}$and $B^{ \pm} \rightarrow K^{+} K^{-} \pi^{ \pm}$[9] finding inclusive charge asymmetries after removing regions dominated by resonances:

$$
\begin{aligned}
& A_{C P}\left(B^{ \pm} \rightarrow \pi^{+} \pi^{-} \pi^{ \pm}\right)= \\
&+ 0.120 \pm 0.020(\text { stat }) \pm 0.019(\text { syst }) \pm 0.007(J / \psi K) \\
& A_{C P}\left(B^{ \pm} \rightarrow K^{+} K^{-} \pi^{ \pm}\right)= \\
& \quad-0.153 \pm 0.046(\text { stat }) \pm 0.019(\text { syst }) \pm 0.007(J / \psi K) \\
& A_{C P}\left(B^{ \pm} \rightarrow \pi^{+} \pi^{-} K^{ \pm}\right)= \\
& \quad 0.032 \pm 0.008(\text { stat }) \pm 0.004(\text { syst }) \pm 0.007(J / \psi K) \\
& A_{C P}\left(B^{ \pm} \rightarrow K^{+} K^{-} K^{ \pm}\right)= \\
&-0.043 \pm 0.009(\text { stat }) \pm 0.003(\text { syst }) \pm 0.007(J / \psi K)
\end{aligned}
$$

where the uncertainties are statistical, systematic and due to the $B^{ \pm} \rightarrow J / \psi K^{ \pm}$mode that is used to cancel the effect of production and detection asymmetries. These results correspond to statistical significances of 4.2, 3.0, 2.8 and 3.7 standard deviations. The charge asymmetries are not uniformly distributed in the phase space and, in addition to the inclusive asymmetries, much larger asymmetries, with significances of more than $9 \sigma$, are observed in localized regions of the phase space.

\section{$3 B_{(s)}^{0} \rightarrow V V^{\prime}$ penguins}

Penguin decays of $B$ mesons are dominated by Feynman diagrams in which a virtual $t$-quark, the heaviest SM elementary particle, is exchanged forming a loop with a $W$ boson. These processes are sensitive probes to the existence of interfering contributions that could be mediated by virtual particles beyond the SM with a mass comparable or higher than that of the $t$-quark.

Because the SM predicts a small CP violating weak phase in the product of CKM matrix elements for loopinduced $b \rightarrow s$ transitions, any observed deviation could indicate physics beyond the SM. For $b \rightarrow d$ transitions the SM branching fraction is suppressed by an order of magnitude with respect $b \rightarrow s$ processes due to a $\left|V_{t d}\right|^{2} /\left|V_{t s}\right|^{2}$ factor, hence they are sensitive to more subtle effects.

The BaBar and Belle experiments have explored $b \rightarrow$ $s q \bar{q}$ and $b \rightarrow d q \bar{q}$ processes into light scalar mesons [10]. Whilst no diminishing the importance of such decays the study of in $B_{(s)}^{0} \rightarrow V V^{\prime}$ penguin modes, where $V$ and $V^{\prime}$ are light vector mesons, are, as it is also for $B^{+}$decays into three bodies, ideal channels to understand the physics of $B$ mesons hadronic decays. There are five modes in this category. Three of them: $B^{0} \rightarrow \phi K^{* 0}$, $B_{s}^{0} \rightarrow \phi \phi$ and $B_{s}^{0} \rightarrow K^{* 0} \bar{K}^{* 0}$ are $b \rightarrow s$ penguins that have been already observed, whereas there are two penguin decay modes that correspond to $b \rightarrow d$ transition loops: the $B_{s}^{0} \rightarrow \phi \bar{K}^{* 0}$ decay, recently discovered [11], and the $B^{0} \rightarrow K^{* 0} \bar{K}^{* 0}$ decay, that remains controversial to the date since the BaBar collaboration reported its discovery with $6 \sigma$ significance and a measurement of its branching fraction of $\mathcal{B}\left(B^{0} \rightarrow K^{* 0} \bar{K}^{* 0}\right)=\left(1.28_{-0.30}^{+0.35} \pm 0.11\right) \times 10^{-6}$ [12] whereas the Belle collaboration established an upper limit of $\mathcal{B}\left(B^{0} \rightarrow K^{* 0} \bar{K}^{* 0}\right)<0.8 \times 10^{-6}$ at the $90 \%$ confidence level [13]. 


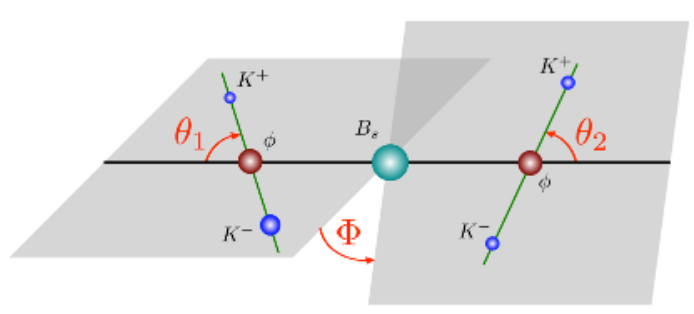

Figure 2. Angles definition for the analysis of $B_{s}^{0} \rightarrow \phi \phi$. The prescription is very similar for the $B_{s}^{0} \rightarrow K^{* 0} \bar{K}^{* 0}$ with $\theta_{1}\left(\theta_{2}\right)$ defined with respect to the kaon direction of the $K^{* 0}\left(\bar{K}^{* 0}\right)$ direction.

\subsection{First measurement of the $\mathrm{CP}$ violating phase in $B_{s}^{0} \rightarrow \phi \phi$ decays}

When the $B_{(s)}^{0}$ and $\bar{B}_{(s)}^{0}$ mesons decay to the same final state there is quantum interference between the direct decay and the decay after a $B_{(s)}^{0}$ oscillation $^{2}$ leading to a CP asymmetry characterised by a CP violating weak phase, $\phi_{s}$. The canonical example is the $B_{s}^{0} \rightarrow J / \psi \phi$ decay. However, other modes, and particularly the penguin decays $B_{s}^{0} \rightarrow K^{* 0} \bar{K}^{* 0}, B^{0} \rightarrow K^{* 0} \bar{K}^{* 0}$ and $B_{s}^{0} \rightarrow \phi \phi$, also experience this effect. In this section the $\mathrm{LHCb}$ result for the latter channel that has been recently published in [14] is reviewed. The value for the $\mathrm{CP}$ violating phase depends on the final state. For the $B_{s}^{0} \rightarrow \phi \phi$ mixing the SM predicts $\phi_{s}$ to be close to zero. Calculations in the QCD factorization approach provide an upper limit of $0.02 \mathrm{rad}$ [16].

The analysis of $B_{s}^{0} \rightarrow \phi \phi$ events involves three helicity angles and the decay time. In the time dependent part, the decay width difference between the light and heavy $B_{s}^{0}$ mass eigenstates, the average decay width and the $B_{s}^{0}$ oscillation frequency are constrained to the values measured by LHCb [17]. The prescription defining the angles is shown in Fig. 2. The probability density function describing the time evolution of the $B_{s}^{0} \rightarrow \phi \phi$ decay depends on six parameters: three polarization fractions $\left|A_{0}\right|^{2},\left|A_{\|}\right|^{2}$ and $\left|A_{\perp}\right|^{2}\left(\left|A_{0}\right|^{2}+\left|A_{\|}\right|^{2}+\left|A_{\perp}\right|^{2}=1\right)$, two strong phases: $\delta_{\|}$ and $\delta_{\perp}$ and the CP violating phase $\phi_{s}$. Additionally an Swave contribution originated from a pair of kaons either in a non-resonant state or from the $f_{0}(980)$ spin- 0 resonance is included in the analysis with amplitude $\left|A_{S}\right|$ and relative strong phase $\delta_{S}$. This $\mathrm{S}$-wave contribution is determined to be $(2.1 \pm 1.2) \%$.

The time dependent study requires knowledge of the $B_{(s)}^{0}$ flavour at production. This is achieved using flavour tagging algorithms [18]. The results of the analysis are shown in Table 1 and Fig. 3. The systematic uncertainties are dominated by time acceptance effects and the determination of the $\mathrm{S}$-wave contribution.

The CP violating phase, $\phi_{s}$, is restricted to the interval $[-2.46,-0.76] \mathrm{rad}$ at $68 \% \mathrm{CL}$. This gives a $16 \%$ $\mathrm{p}$-value for the prediction of [15], taking the values of the strong phases and polarization amplitudes observed in data and assuming that systematic uncertainties are negligible. The result of the angular polarization fractions does

\footnotetext{
${ }^{2}$ As a consequence of flavour changing neutral currents $B_{(s)}^{0}$ mesons oscillate back a forth into $\bar{B}_{(s)}^{0}$ mesons.
}

Table 1. Fit results with statistical and systematic uncertainties. A $68 \%$ statistical confidence interval is quoted for $\phi_{s}$. Amplitudes are defined at $t=0$.

\begin{tabular}{lccc}
\hline Parameter & Value & $\sigma_{\text {stat. }}$ & $\sigma_{\text {syst. }}$ \\
\hline$\phi_{s}[\mathrm{rad}](68 \% \mathrm{CL})$ & & {$[-2.37,-0.92]$} & 0.22 \\
$\left|A_{0}\right|^{2}$ & 0.329 & 0.033 & 0.017 \\
$\left|A_{\perp}\right|^{2}$ & 0.358 & 0.046 & 0.018 \\
$\left|A_{\mathrm{S}}\right|^{2}$ & 0.016 & +0.024 & 0.009 \\
$\delta_{\perp}-\delta_{\|}[\mathrm{rad}]$ & 2.19 & -0.012 & 0.12 \\
$\delta_{\perp}-\delta_{0}[\mathrm{rad}]$ & -1.47 & 0.44 & 0.10 \\
$\delta_{\mathrm{S}}[\mathrm{rad}]$ & 0.65 & +0.89 & 0.33 \\
\hline
\end{tabular}

not superseed the longitudinal polarization value $\left|A_{0}\right|^{2}=$ $0.365 \pm 0.025$ [19], previously obtained in LHCb performing an untagged time integrated analysis of the channel.

\subsection{First observation of the $B_{s}^{0} \rightarrow \phi \bar{K}^{* 0}$ decay}

From the V-A structure of the weak interaction and helicity conservation in the strong interaction, the final state of $B_{(s)}^{0} \rightarrow V V^{\prime}$ decays is expected to be highly longitudinally polarized. This applies to both tree and penguin decays. The BaBar and Belle experiments have confirmed that longitudinal polarization dominates in $b \rightarrow u$ tree level processes [20]. However, analyses of the polarization in decays with both tree and penguin contributions, such as $B^{0} \rightarrow \rho^{0} K^{* 0}$ and $B^{0} \rightarrow \rho^{-} K^{*+}$ [21] and in $b \rightarrow s$ penguin decays, $B^{0} \rightarrow \phi K^{* 0}[22], B_{s}^{0} \rightarrow K^{* 0} \bar{K}^{* 0}$ [23] and $B_{s}^{0} \rightarrow \phi \phi[14,19,24]$, indicate a low value of the longitudinal polarization fraction comparable with, or even smaller than, the transverse fraction. Table 2 gives a summary of the longitudinal polarization fractions displaying the diversity of results and the nature of the decay channels. In view of these results, the discovery and study of the angular parameters of the $B_{s}^{0} \rightarrow \phi \bar{K}^{* 0}$ decay is crucial to provide input that helps understanding this, sometimes called, "polarization puzzle".

The analysis is performed with the 2011 data collected by the LHCb experiment. The signal discrimination consists of requiring invariant masses of $K^{-} \pi^{+}$and $K^{+} K^{-}$ pairs compatible with the known masses of the $\bar{K}^{* 0}$ and $\phi$ resonances. In addition, a selection on the four final particles impact parameters (IP), transverse momenta $\left(p_{t}\right)$ and particle identification in the RICH has been applied. The geometrical characteristics of the $B_{s}^{0}$ meson candidates, such as their IP, decay time, the minimum $\chi_{I P}^{2}$ of the four tracks with respect to all primary vertices in the event and the distance of closest approach between the $\bar{K}^{* 0}$ and $\phi$ candidates' trajectories, as well as their $p_{t}$ are combined in a single discriminating variable using a Geometrical Likelihood method [25]. The result of the selection produces 1277 candidates in the four-body mass range from 4915 to $5815 \mathrm{MeV} / \mathrm{c}^{2}$.

The four-body mass spectrum of these events is parametrized accounting for five main contributions: the $B_{s}^{0} \rightarrow \phi \bar{K}^{* 0}$ and $B^{0} \rightarrow \phi K^{* 0}$ decays, partially reconstructed decays of $B$ mesons, combinatorial background and a small fraction of $\Lambda_{b}^{0}$ decays where the proton has been misidentified as a kaon or a pion. The fit result gives 

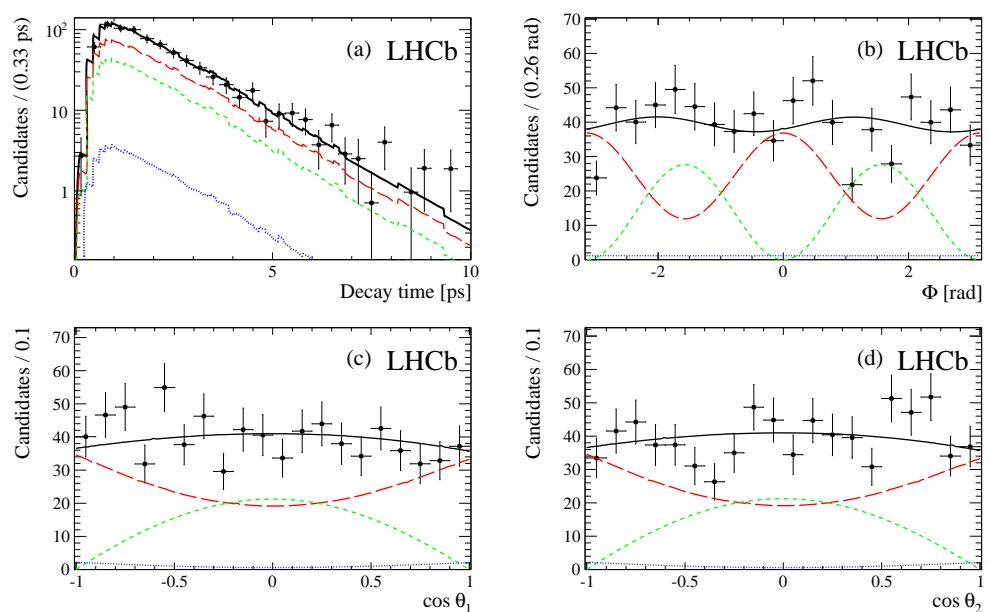

Figure 3. One-dimensional projections of the $B_{s}^{0} \rightarrow \phi \phi$ fit for (a) decay time, (b) helicity angle $\Phi$ and the cosine of the helicity angles (c) $\theta_{1}$ and (d) $\theta_{2}$. The data are marked as points, while the solid lines represent the projections of the best fit. The CP even P-wave, the $\mathrm{CP}$-odd $\mathrm{P}$-wave and $\mathrm{S}$-wave components are shown by the long dashed, short dashed and dotted lines, respectively.

Table 2. Summary of experimental results on longitudinal polarization fractions for a representative Tree dominated decay, mode, a decay mode with significant Tree and Penguin contributions and the pure penguin $B_{(s)}^{0} \rightarrow V V^{\prime}$ decays.

\begin{tabular}{cccccccc}
\hline Mode & $B^{0} \rightarrow \rho^{+} \rho^{-}$ & $B^{0} \rightarrow \rho^{0} K^{* 0}$ & $B_{s}^{0} \rightarrow \phi \phi$ & $B_{s}^{0} \rightarrow K^{* 0} \bar{K}^{* 0}$ & $B^{0} \rightarrow K^{* 0} \bar{K}^{* 0}$ & $B^{0} \rightarrow \phi K^{* 0}$ & $B_{s}^{0} \rightarrow \phi \bar{K}^{* 0}$ \\
Type and Ref. & Tree [26] & Tree+Penguin [21] & Penguin [19] & Penguin [23] & Penguin [12] & Penguin [22] & Penguin [11] \\
\hline$\left|A_{0}\right|^{2}$ & $0.977 \pm 0.028$ & $0.40 \pm 0.14$ & $0.365 \pm 0.025$ & $0.31 \pm 0.13$ & $0.80 \pm 0.13$ & $0.494 \pm 0.036$ & $0.51 \pm 0.17$ \\
\hline
\end{tabular}

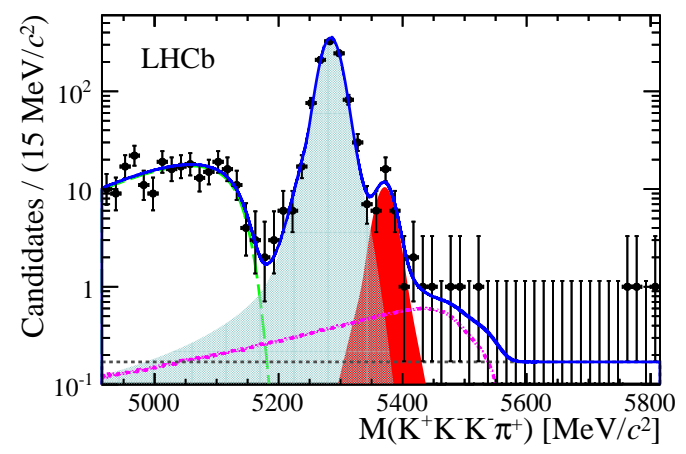

Figure 4. Four-body $K^{+} K^{-} K^{-} \pi^{+}$invariant mass distribution. The points show the data, the blue solid line shows the overall fit, the solid dark red shaded region is the $B_{s}^{0} \rightarrow \phi \bar{K}^{* 0}$ signal, the light blue shaded region corresponds to the $B^{0} \rightarrow \phi K^{* 0}$ signal, the grey dotted line is the combinatorial background and the green dashed line and magenta dashed-dotted lines are the partially reconstructed and misidentified $\Lambda_{b}^{0}$ backgrounds.

a total of $30 \pm 6 B_{s}^{0} \rightarrow\left(K^{+} K^{-}\right)\left(K^{-} \pi^{+}\right)$candidates within the mass windows $1012.5<M\left(K^{+} K^{-}\right)<1026.5 \mathrm{MeV} / c^{2}$ and $746<M\left(K^{-} \pi^{+}\right)<1046 \mathrm{MeV} / c^{2}$. The result translates into an observation with a significance of $6.1 \sigma$ for the $B_{s}^{0} \rightarrow \phi \bar{K}^{* 0}$ signal taking into account $\mathrm{S}$-wave contributions. The analysis of the $K^{+} K^{-}$and the $K^{-} \pi^{+}$mass distributions is consistent with $(84 \pm 2) \%$ of the signal originating from resonant $\phi$ and $\bar{K}^{* 0}$ mesons.

As mentioned, the $B_{s}^{0} \rightarrow \phi \bar{K}^{* 0}$ mode is a $b \rightarrow d$ penguin decay that has not previously been observed. It
Table 3. Results of the fit to the sample of selected candidates.

\begin{tabular}{cc}
\hline Contribution & Yield \\
\hline$B_{s}^{0} \rightarrow \phi \bar{K}^{* 0}$ & $30 \pm 6$ \\
$B^{0} \rightarrow \phi K^{* 0}$ & $1000 \pm 32$ \\
Partially reconstructed background & $218 \pm 15$ \\
$\Lambda_{b}^{0}$ background & $13 \pm 8$ \\
Combinatorial background & $10 \pm 6$ \\
\hline
\end{tabular}

only differs from the $B^{0} \rightarrow \phi K^{* 0}$ decay in the spectator quark and the final quark in the loop, therefore the relative branching fractions of these two channels should scale as $\left|V_{t d}\right|^{2} /\left|V_{t s}\right|^{2} 3$ and their polarization fractions are expected to be very similar.

Since both decays share the same final state, except for charge conjugation, $B^{0} \rightarrow \phi K^{* 0}$ is the ideal normalization channel for the determination of the $B_{s}^{0} \rightarrow \phi \bar{K}^{* 0}$ branching fraction. However, the two decay channels have slightly different polarizations, as explained below, and consequently their angular distributions differ. This affects the relative efficiencies of the two channels and is accounted for in the form of a tiny correction $\lambda=1.01 \pm 0.06$.

With the branching fraction $\mathcal{B}\left(B^{0} \rightarrow \phi K^{* 0}\right)=(9.8 \pm$ $0.6) \times 10^{-6}$ [27] and the number of $B_{s}^{0} \rightarrow \phi \bar{K}^{* 0}\left(N_{B_{s}^{0} \rightarrow \phi \bar{K}^{* 0}}\right)$ and $B^{0} \rightarrow \phi K^{* 0}\left(N_{B^{0} \rightarrow \phi K^{* 0}}\right)$ candidates, the branching fraction of the $B_{s}^{0} \rightarrow \phi \bar{K}^{* 0}$ decay is computed as

$$
\mathcal{B}\left(B_{s}^{0} \rightarrow \phi \bar{K}^{* 0}\right)=\lambda \times \frac{f_{d}}{f_{s}} \times \mathcal{B}\left(B^{0} \rightarrow \phi K^{* 0}\right) \times \frac{N_{B_{s}^{0} \rightarrow \phi \bar{K}^{* 0}}}{N_{B^{0} \rightarrow \phi K^{* 0}}}
$$

\footnotetext{
${ }^{3} \mid V_{t d}$ and $V_{t s}$ are CKM matrix elements and their ratio is known to be $\left|V_{t d}\right| /\left|V_{t s}\right|=0.211 \pm 0.006[27]$.
} 
where $f_{d} / f_{s}=3.75 \pm 0.29$ is the ratio of hadronization factors measured by $\mathrm{LHCb}$ and needed to account for the different production rates of $B^{0}$ and $B_{s}^{0}$ mesons [28]. This translates into the final result of

$$
\begin{aligned}
& \mathcal{B}\left(B_{s}^{0} \rightarrow \phi \bar{K}^{* 0}\right)= \\
& \left(1.10 \pm 0.24 \text { (stat) } \pm 0.14 \text { (syst) } \pm 0.08\left(\frac{f_{d}}{f_{s}}\right)\right) \times 10^{-6}
\end{aligned}
$$

The four main sources of systematic uncertainty were considered to be: the fit model, the dependence of the acceptance on the longitudinal polarization, the purity of the signal and the uncertainty in the relative efficiency of $B_{s}^{0}$ and $B^{0}$ detection.

Besides of the branching fraction determination, a flavour-averaged and time-integrated polarization analysis is performed assuming the $\mathrm{CP}$ violating phase to be zero and $B_{s}^{0}$ and $\bar{B}_{s}^{0}$ mesons produced with the same fraction. With the same angle prescription of Fig. 2, discussed for the analysis of $B_{s}^{0} \rightarrow \phi \phi$ decays, the polarization amplitudes $\left|A_{0}\right|$ and $\left|A_{\|}\right|$and the strong phase cosine, $\cos \delta_{\|}$, are accessible.

The angular fit to data in $\mathrm{a} \pm 30 \mathrm{MeV} / \mathrm{c}^{2}$ window around the $B_{s}^{0}$ mass gives the results

$$
\begin{aligned}
\left|A_{0}\right|^{2} & =0.51 \pm 0.15(\text { stat }) \pm 0.07 \text { (syst) } \\
\left|A_{\|}\right|^{2} & =0.21 \pm 0.11 \text { (stat) } \pm 0.02(\text { syst }) \\
\cos \delta_{\|} & =-0.18 \pm 0.52 \text { (stat) } \pm 0.29 \text { (syst) }
\end{aligned}
$$

where contamination of decays from $B^{0} \rightarrow \phi K^{* 0}$ crossfeed, $\Lambda_{b}^{0}$ decays and combinatorial background are accounted for. The source of systematic effects considered are: the uncertainties in the spectrometer acceptance, the combinatorial background angular distribution and the $S$ wave fraction (whose central value is fixed to the same amount as for the $B^{0} \rightarrow \phi K^{* 0}$ decay).

The final result of the longitudinal polarization fraction contradicts the naïve expectation of large values, but is consistent with the values of the $b \rightarrow s$ penguin decays. The result is compatible with the longitudinal polarization fraction $\left|A_{0}\right|^{2}=0.40 \pm 0.14$ measured in the $B^{0} \rightarrow \rho^{0} K^{* 0}$ decay [21], the penguin amplitude of which is related to $B_{s}^{0} \rightarrow \phi \bar{K}^{* 0}$ by $d \leftrightarrow s$ exchange. Finally, $\left|A_{0}\right|^{2}$ is smaller than the prediction of perturbative QCD, 0.712 ${ }_{-0.048}^{+0.042}$ [29].

\section{Summary}

In the context of charmless $B$ decays studies in the $\mathrm{LHCb}$ experiment several results have been summarized. The first observation of CP violation in $B_{s}^{0}$ decays and an improvement in the determination of the $A_{C P}\left(B^{0} \rightarrow K^{+} \pi^{-}\right)$ have been provided. The observation of direct $\mathrm{CP}$ violation in 3-body decays has also been reviewed. The first time dependent analysis in $B_{s}^{0} \rightarrow \phi \phi$ decays, leading to a $68 \%$ confidence level for the $\mathrm{CP}$ violating phase $\phi_{s}$ of $[-2.46,-0.76] \mathrm{rad}$ has been presented. Finally, the first observation of the $B_{s}^{0} \rightarrow \phi \bar{K}^{* 0}$ decay has been reported together with an untagged time integrated analysis of its angular distributions.

\section{References}

[1] N. Cabibbo, Phys. Rev. Lett. 10 531. M. Kobayashi and T. Maskawa, Prog. Theor. Phys. 49652.

[2] R. Aaij et al., Phys. Lett. B 694209.

[3] R. Aaij et al., LHCb-CONF-2010-013.

[4] A. A. Alves, Jr. et al., JINST 3 S08005.

[5] B. Aubert et al., Phys. Rev. Lett. 93 131801. Y. Chao et al., Phys. Rev. D 71031502.

[6] RAaij et al., Phys. Rev. Lett. 110221601.

[7] H. J. Lipkin, Phys. Lett. B 621126.

[8] RAaij et al., LHCb-CONF-2012-028.

[9] RAaij et al., arXiv:1306.1246 [hep-ex].

[10] K. Abe et al., Phys. Rev. Lett. 91 261602. B. Aubert et al., Phys. Rev. Lett. 94 191802. B. Aubert et al., Phys. Rev. D 71091102.

[11] RAaij et al., arXiv:1306.2239 [hep-ex].

[12] B. Aubert et al., Phys. Rev. Lett. 100081801.

[13] C.-C. Chiang et al., Phys. Rev. D 81071101.

[14] RAaij et al., Phys. Rev. Lett. 110, 241802.

[15] M. Raidal, Phys. Rev. Lett. 89231803.

[16] M. Bartsch, G. Buchalla and C. Kraus, arXiv:0810.0249 [hep-ph]. M. Beneke, J. Rohrer and D. Yang, Nucl. Phys. B 774 64. H.-Y. Cheng and C.-K. Chua, Phys. Rev. D 80114026.

[17] RAaij et al., Phys. Rev. D 87, 112010. RAaij et al., LHCb-CONF-2011-050.

[18] R. Aaij et al., Eur. Phys. J. C 72 2022. RAaij et al., LHCb-CONF-2012.

[19] RAaij et al., Phys. Lett. B 713369.

[20] A. Somov et al., Phys. Rev. D 76 011104. B. Aubert et al., Phys. Rev. D 76 052007. J. Zhang et al., Phys. Rev. Lett. 91 221801. B. Aubert et al., Phys. Rev. Lett. 102 141802. B. Aubert et al., Phys. Rev. D 79, 052005.

[21] J. P. Lees et al., Phys. Rev. D 85072005.

[22] K.-F. Chen et al., Phys. Rev. Lett. 94, 221804. B. Aubert et al., Phys. Rev. D 78092008.

[23] R. Aaij et al., Phys. Lett. B 70950.

[24] T. Aaltonen et al., Phys. Rev. Lett. 107261802.

[25] D. Karlen, Comput. Phys. 12 380. D. Martinez Santos, CERN-THESIS-2010-068.

[26] B. Aubert et al., hep-ex/0607098.

[27] J. Beringer et al., Phys. Rev. D 86010001.

[28] R. Aaij et al., Phys. Rev. Lett. 107211801.

[29] A. Ali, G. Kramer, Y. Li, C. -D. Lu, Y. -L. Shen, W. Wang and Y. -M. Wang, Phys. Rev. D 76074018. 